\title{
Chemoselective Ring Closure of 4-(3-Methyl-2-oxo-2,5-dihydro-1H-pyrrol-1-yl)butanal Leading to Pandalizine A
}

Mahesh B. Yadav, ${ }^{\dagger,}$ Kailas R. Pandhade ${ }^{\dagger, \S}$ and Narshinha P. Argade ${ }^{* \dagger, \S}$

${ }^{\dagger}$ Division of Organic Chemistry, National Chemical Laboratory (CSIR), Pune 411 008, India

${ }^{\S}$ Academy of Scientific and Innovative Research (AcSIR), New Delhi 110 025, India

E-mail: np.argade@ncl.res.in

\begin{tabular}{|c|c|}
\hline Figure number & Contents \\
\hline Figure S1 & ${ }^{1} \mathrm{H}$ NMR spectrum of compound 2a \\
\hline Figure S2 & ${ }^{13} \mathrm{C}$ NMR spectrum of compound 2a \\
\hline Figure S3 & ${ }^{1} \mathrm{H}$ NMR spectrum of compound 3a \\
\hline Figure S4 & ${ }^{13} \mathrm{C}$ NMR spectrum of compound 3a \\
\hline Figure S5 & ${ }^{1} \mathrm{H}$ NMR spectrum of compound 4a \\
\hline Figure S6 & ${ }^{13} \mathrm{C}$ NMR spectrum of compound 4a \\
\hline Figure S7 & ${ }^{1} \mathrm{H}$ NMR spectrum of compound 5a \\
\hline Figure S8 & ${ }^{13} \mathrm{C}$ NMR spectrum of compound 5a \\
\hline
\end{tabular}




\begin{tabular}{|c|c|}
\hline Figure S9 & ${ }^{1} \mathrm{H}$ NMR spectrum of compound 7a \\
\hline Figure S10 & ${ }^{13}$ C NMR spectrum of compound $\mathbf{7 a}$ \\
\hline Figure S11 & ${ }^{1}$ H NMR spectrum of compound $\mathbf{2 b}$ \\
\hline Figure S12 & ${ }^{13}$ C NMR spectrum of compound $\mathbf{2 b}$ \\
\hline Figure S13 & DEPT NMR spectrum of compound $\mathbf{2 b}$ \\
\hline Figure S14 & ${ }^{1} \mathrm{H}$ NMR spectrum of compound $\mathbf{3 b}$ \\
\hline Figure S15 & ${ }^{13}$ C NMR spectrum of compound $\mathbf{3 b}$ \\
\hline Figure S16 & DEPT NMR spectrum of compound 3b \\
\hline Figure S17 & ${ }^{1} \mathrm{H}$ NMR spectrum of compound $\mathbf{4 b}$ \\
\hline Figure S18 & ${ }^{13} \mathrm{C}$ NMR spectrum of compound $\mathbf{4 b}$ \\
\hline Figure S19 & DEPT NMR spectrum of compound $\mathbf{4 b}$ \\
\hline Figure S20 & ${ }^{1} \mathrm{H}$ NMR spectrum of compound $\mathbf{5 b}$ \\
\hline Figure S21 & ${ }^{13} \mathrm{C}$ NMR spectrum of compound $\mathbf{5 b}$ \\
\hline Figure S22 & DEPT NMR spectrum of compound $\mathbf{5 b}$ \\
\hline Figure S23 & ${ }^{1} \mathrm{H}$ NMR spectrum of compound 7b \\
\hline Figure S24 & ${ }^{1} \mathrm{H}$ NMR spectrum of compound 7b \\
\hline Figure S25 & ${ }^{13} \mathrm{C}$ NMR spectrum of compound 7b \\
\hline Figure S26 & DEPT NMR spectrum of compound 7b \\
\hline
\end{tabular}




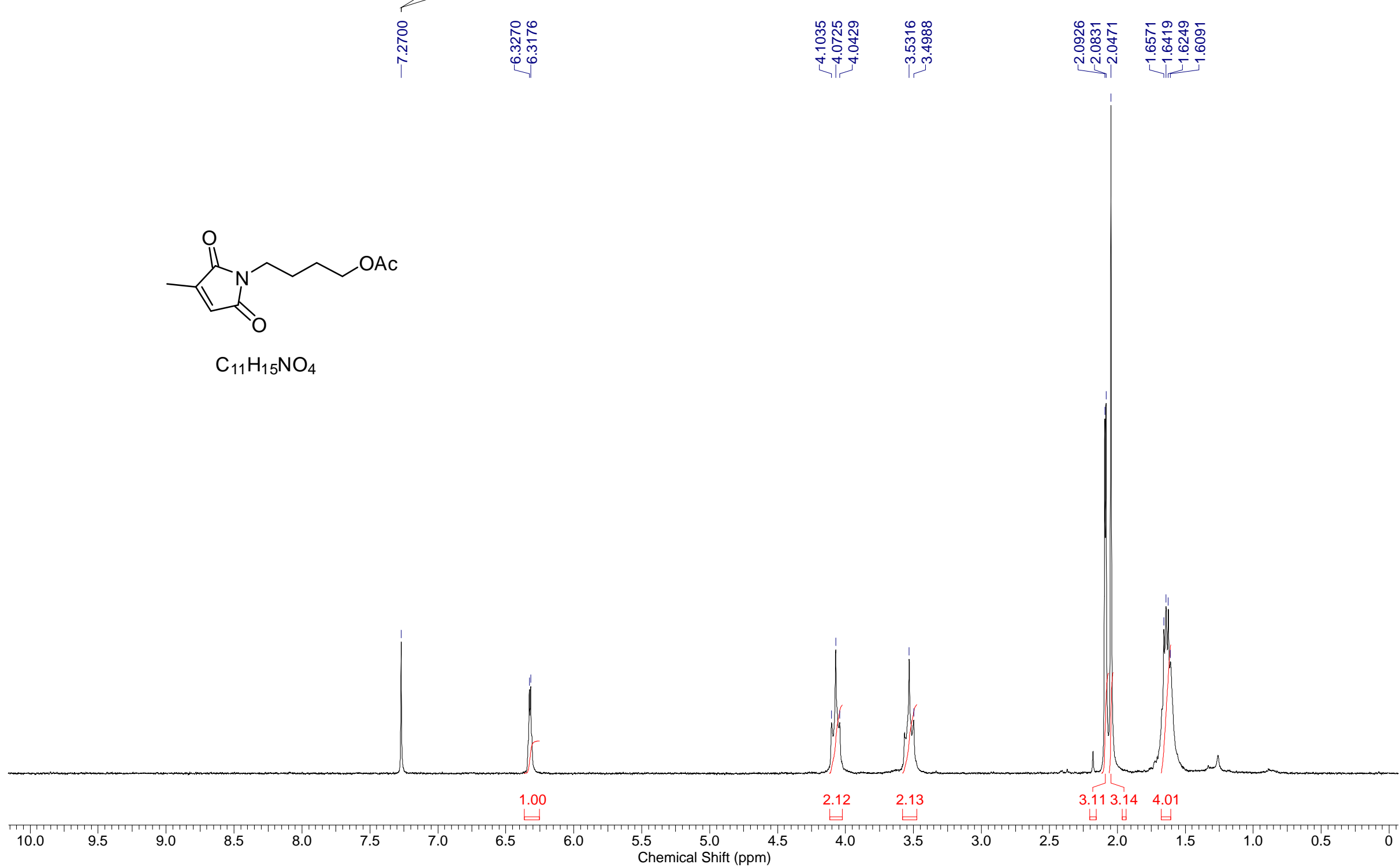




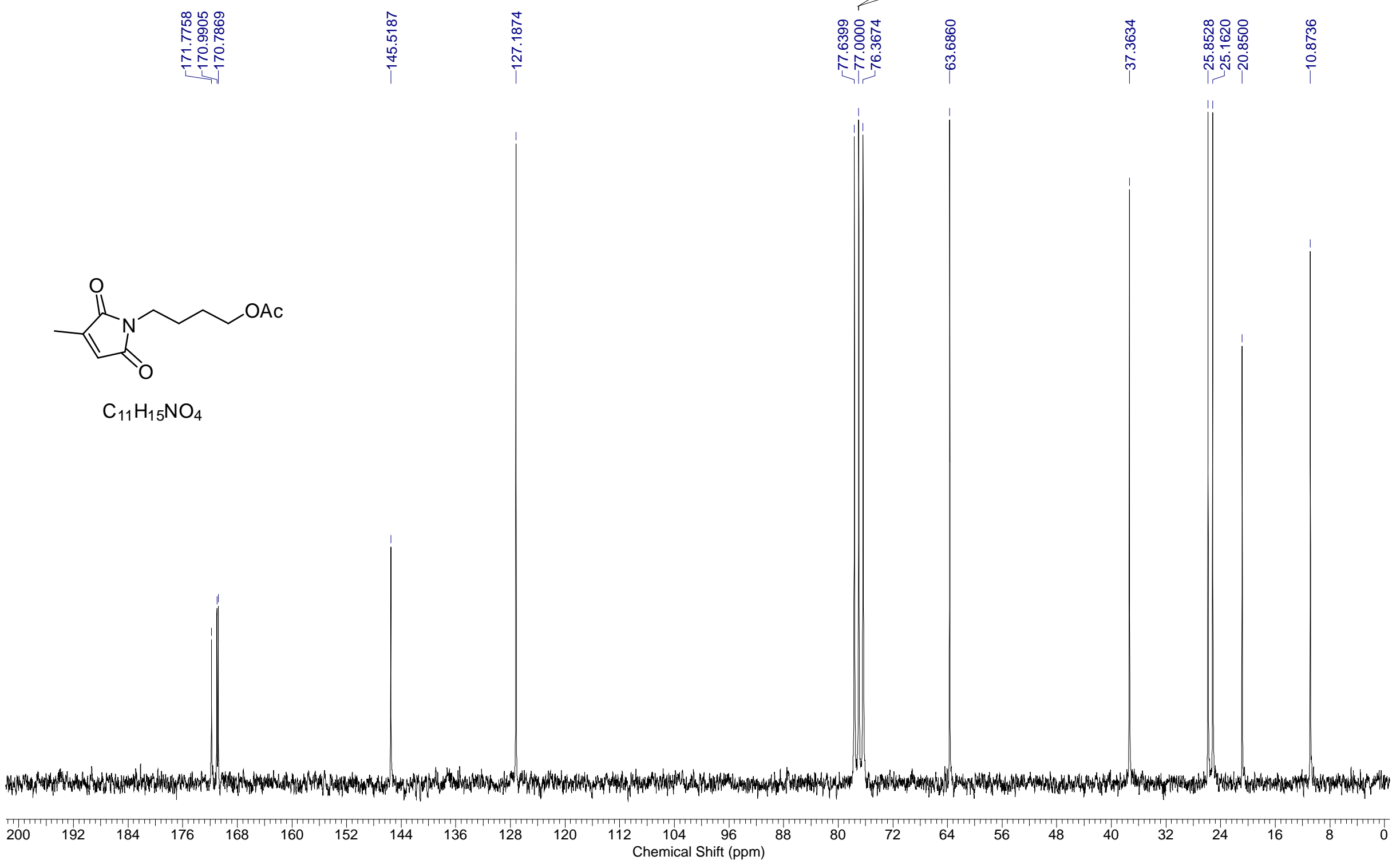




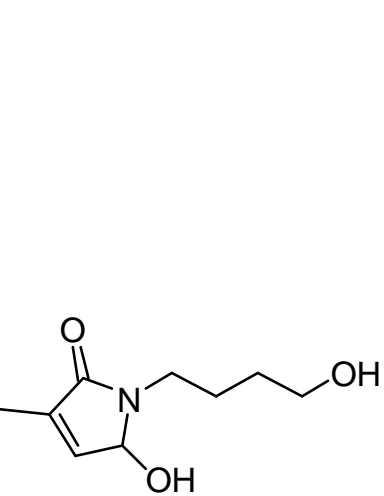

$\mathrm{C}_{9} \mathrm{H}_{15} \mathrm{NO}_{3}$ 


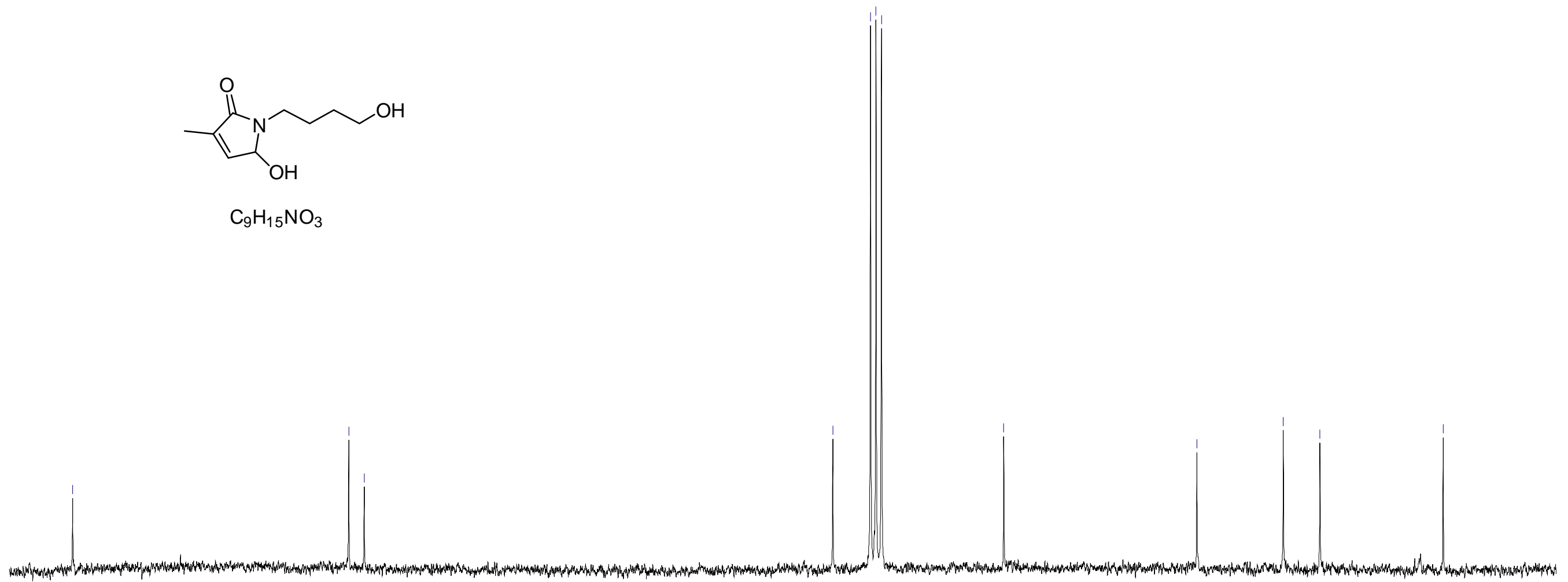




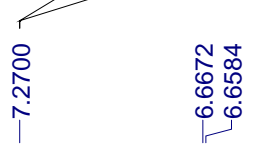

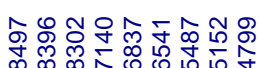

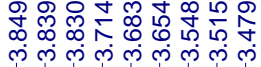

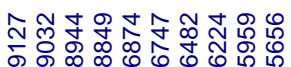

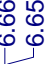

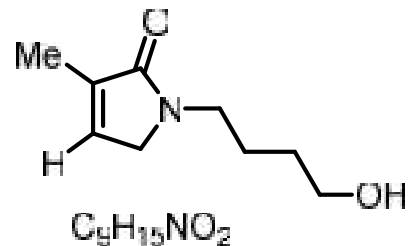

$\mathrm{C}_{\mathrm{y}} \mathrm{H}_{15} \mathrm{NO}_{2}$

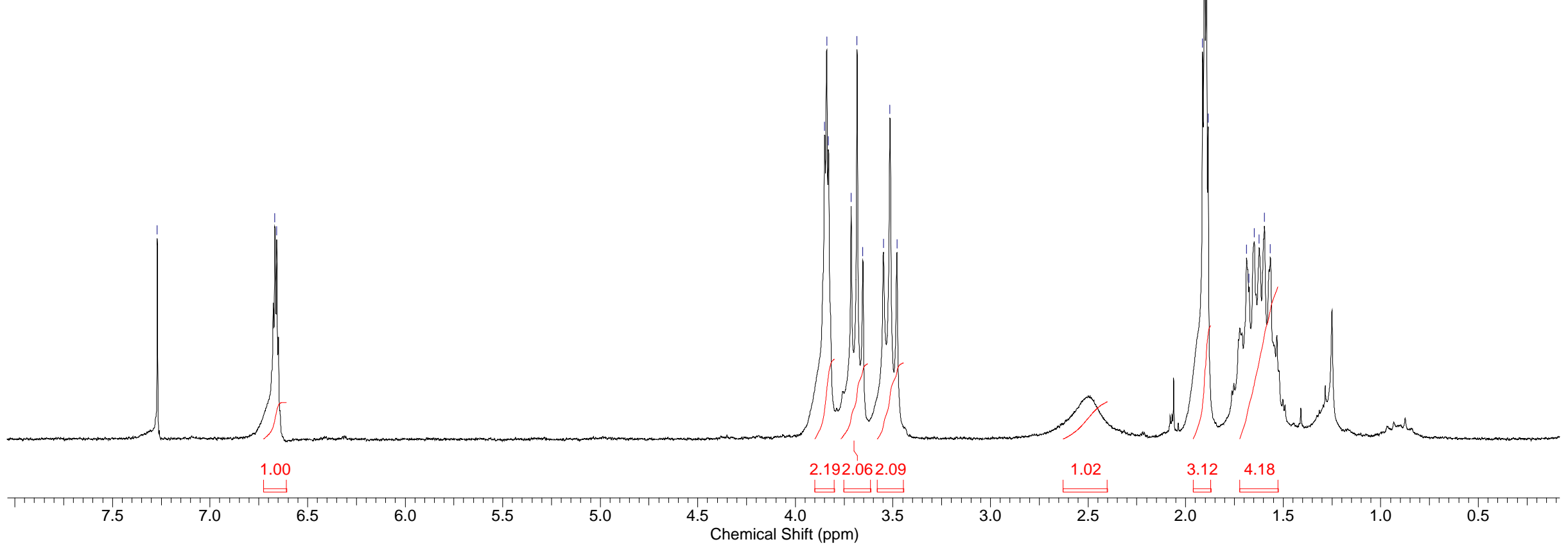




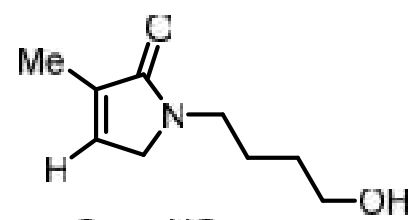

$\mathrm{C}_{4} \mathrm{H}_{15} \mathrm{NO}_{2}$

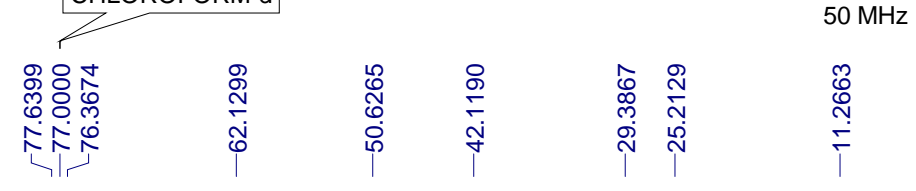

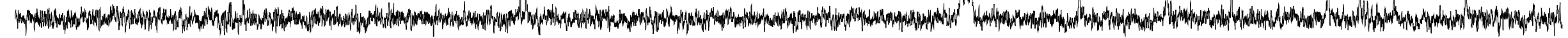




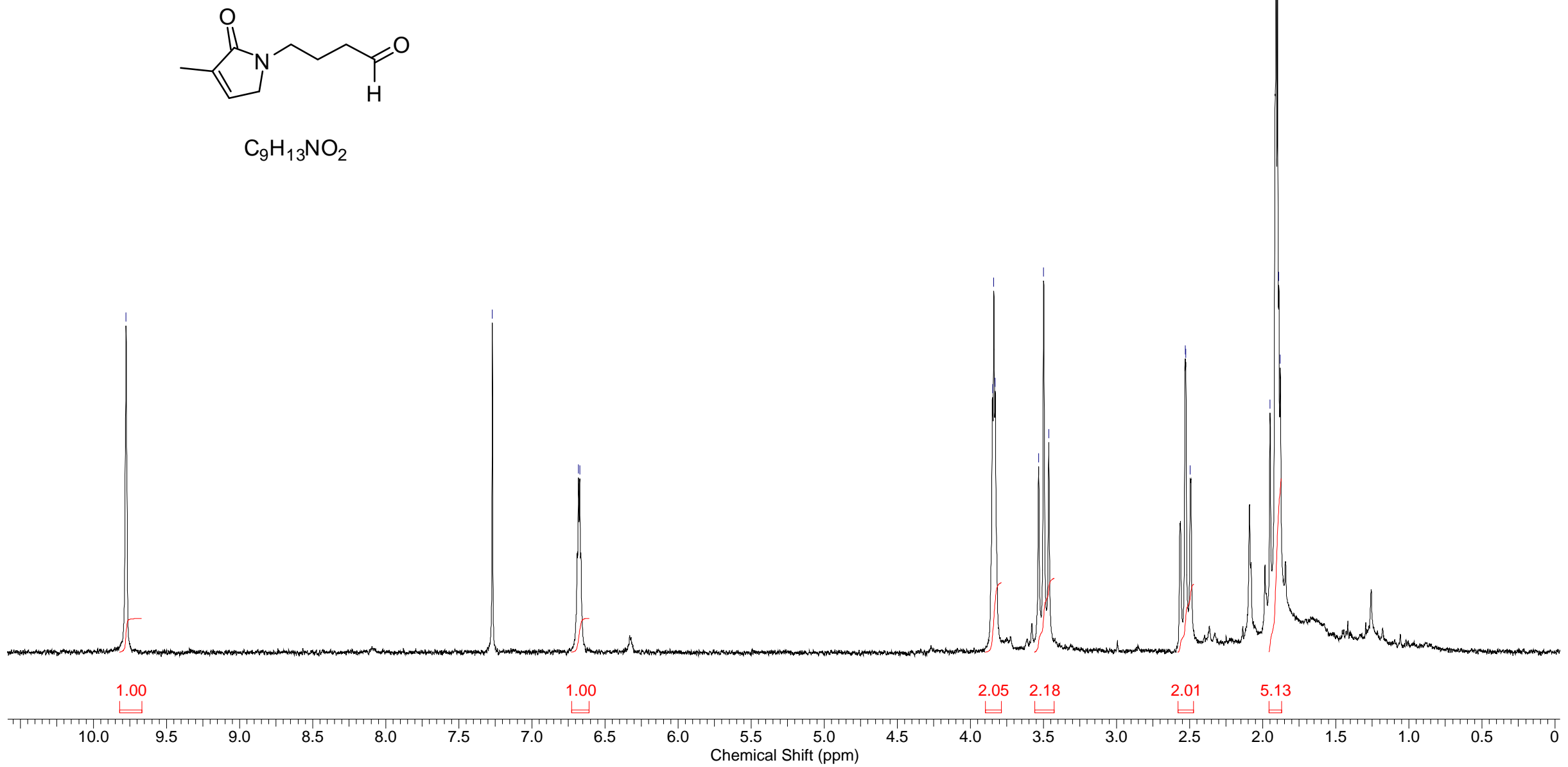

$\mathrm{C}_{9} \mathrm{H}_{13} \mathrm{NO}_{2}$ 
AV-400-20180712-150609-11780.003.001.1r.esp

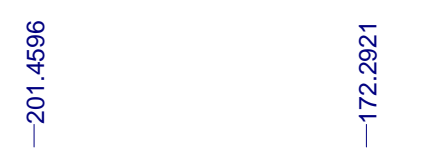

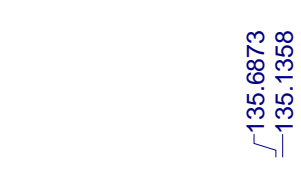

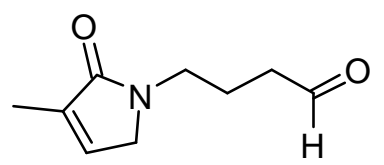

$\mathrm{C}_{9} \mathrm{H}_{13} \mathrm{NO}_{2}$
CHLOROFORM-d

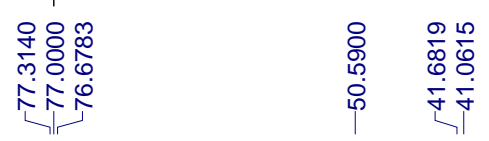

$100 \mathrm{MHz}$

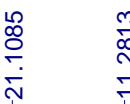

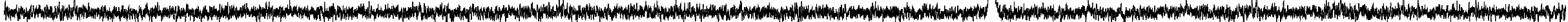

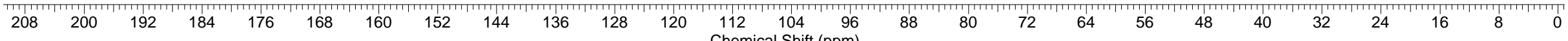


CHLOROFORM-d

\begin{tabular}{|c|c|c|c|}
\hline 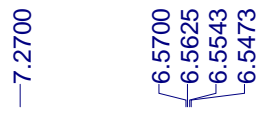 & 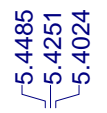 & 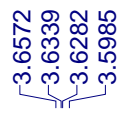 & 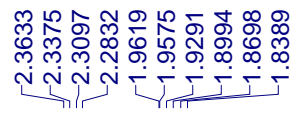 \\
\hline
\end{tabular}

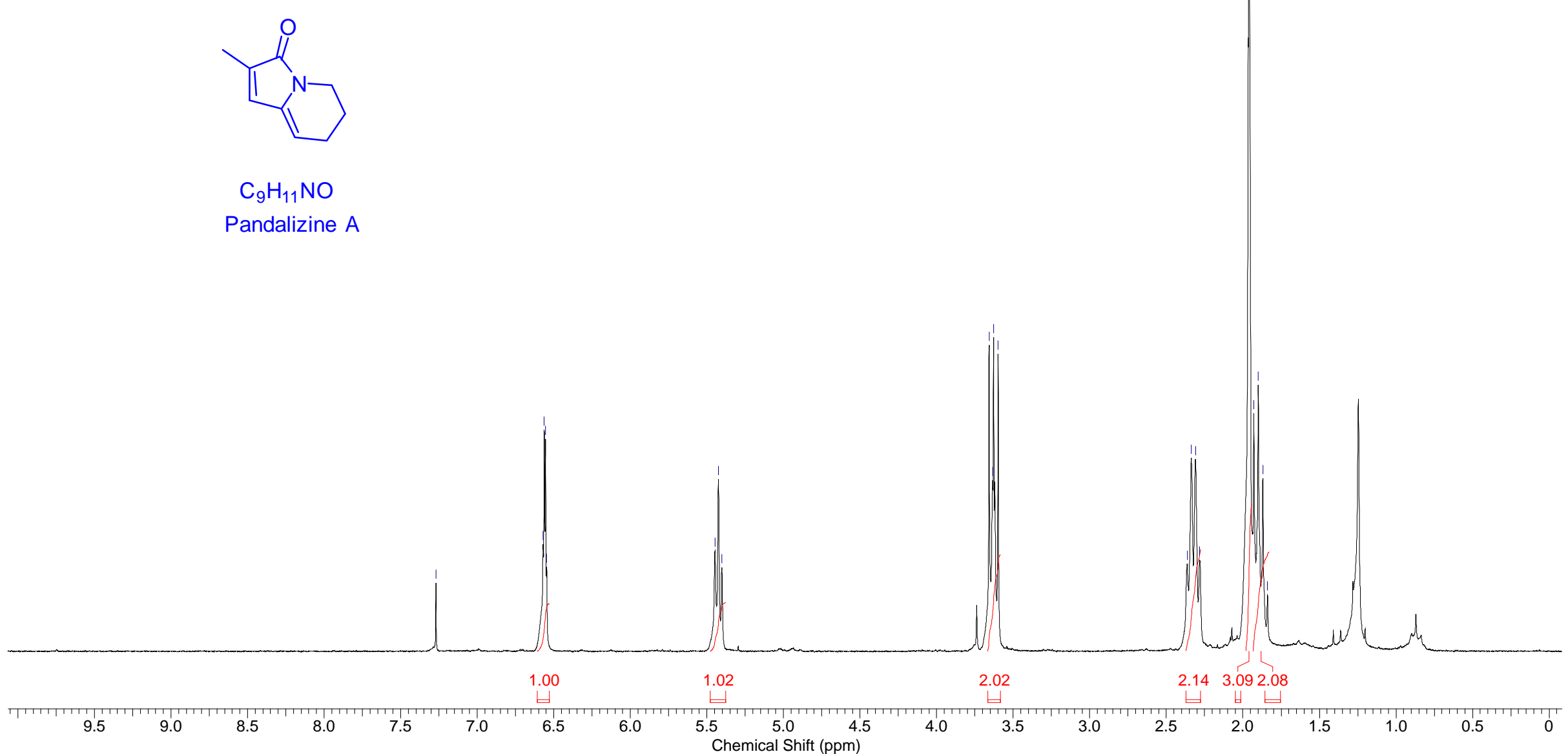

$\mathrm{C}_{9} \mathrm{H}_{11} \mathrm{NO}$

Pandalizine $\mathrm{A}$ 


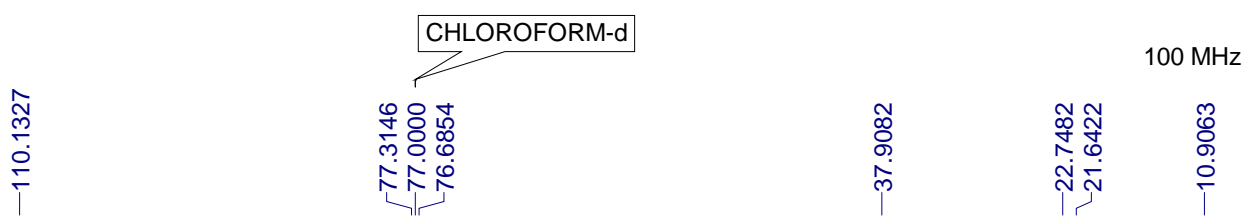

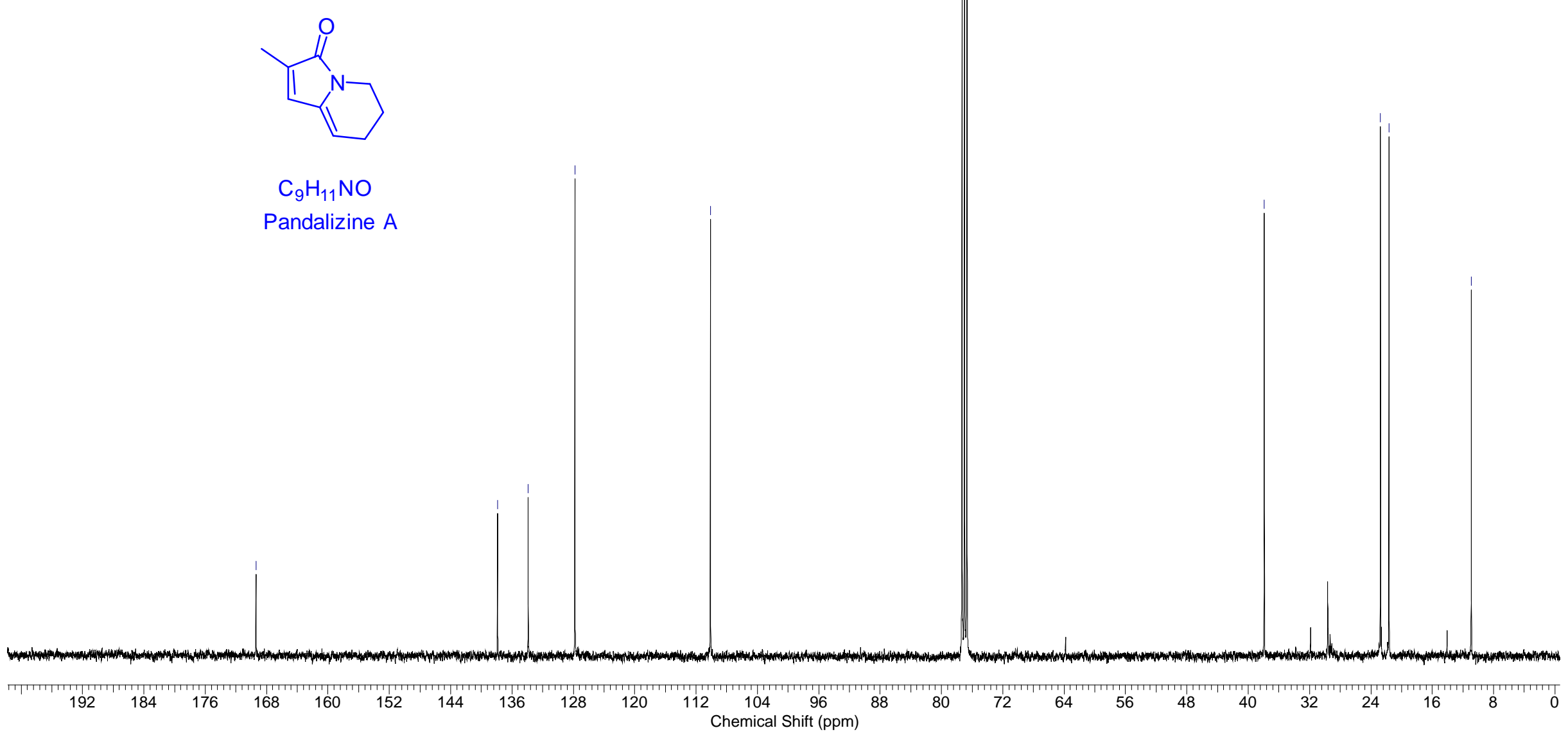

$\mathrm{C}_{9} \mathrm{H}_{11} \mathrm{NO}$

Pandalizine A

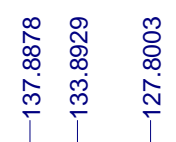
$\underset{\substack{0 \\ 0}}{\substack{0 \\ \sim}}$

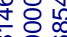
ㅅำ 


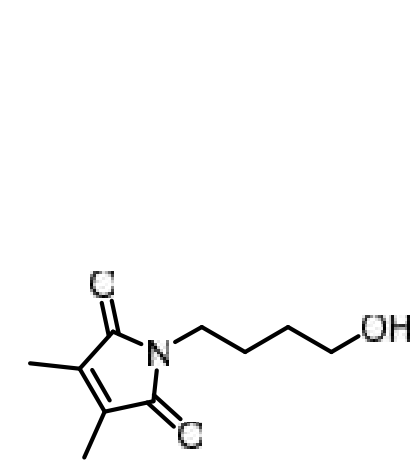

$\mathrm{C}_{10} \mathrm{H}_{15} \mathrm{NO}_{3}$

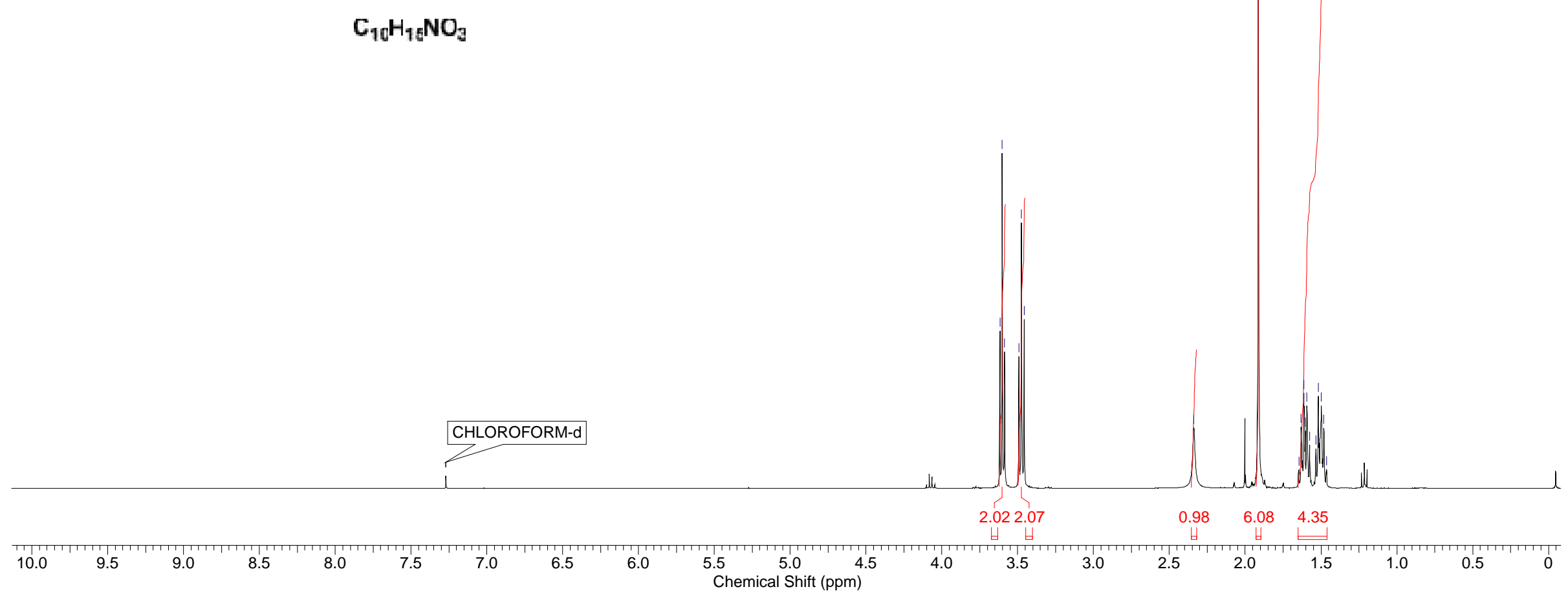




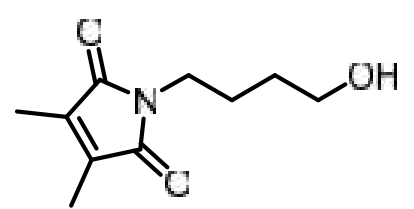

$\mathrm{C}_{10} \mathrm{H}_{15} \mathrm{NO}_{3}$

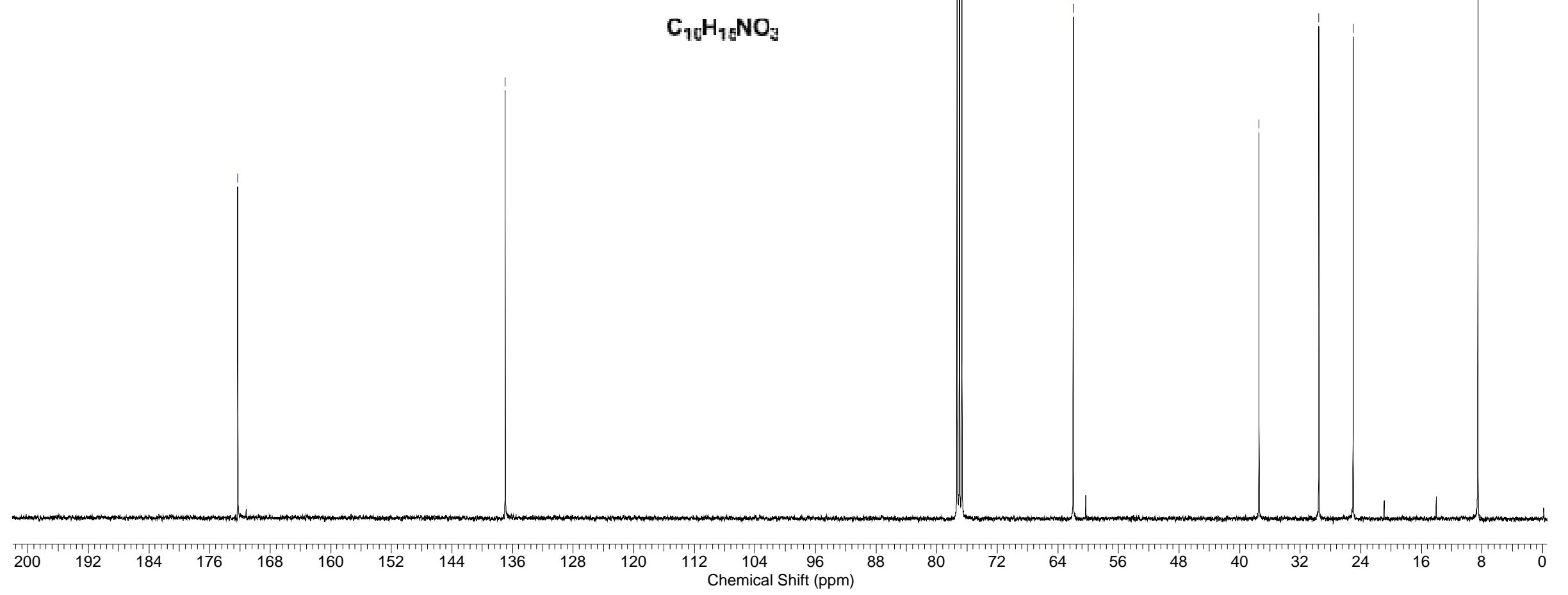

S14

Figure $\mathrm{S} 12:{ }^{13} \mathrm{C}$ NMR spectrum of compound $\mathbf{2 b}$ 


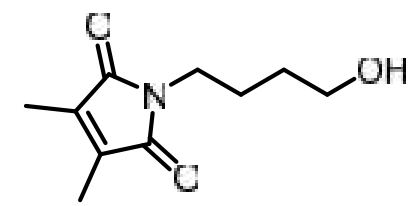

$\mathrm{C}_{10} \mathrm{H}_{15} \mathrm{NO}_{3}$

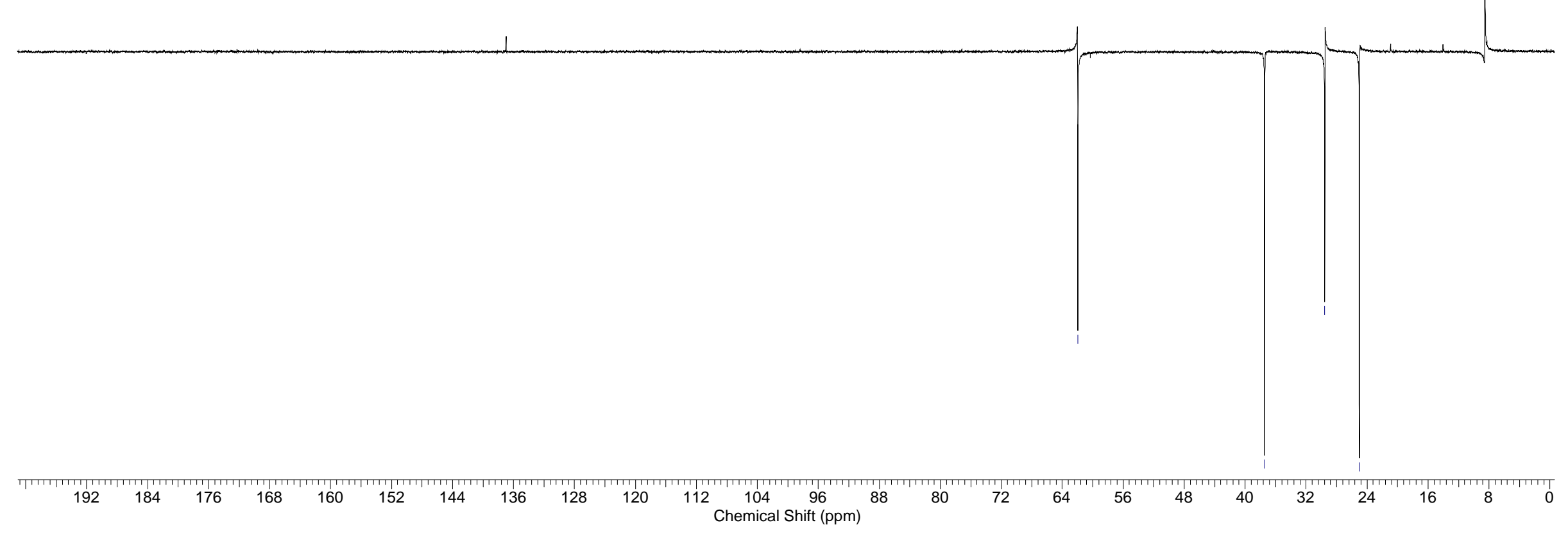




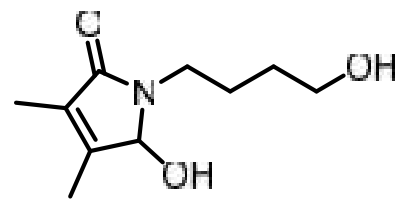

$\mathrm{C}_{10} \mathrm{H}_{17} \mathrm{NO}_{3}$ 


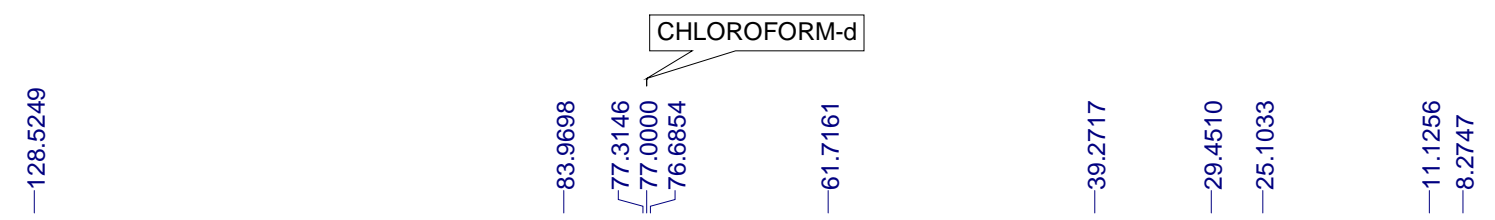<smiles>CC1=C(C)C(O)N(CCCCO)C1=O</smiles>

$\mathrm{C}_{10} \mathrm{H}_{17} \mathrm{NO}_{3}$

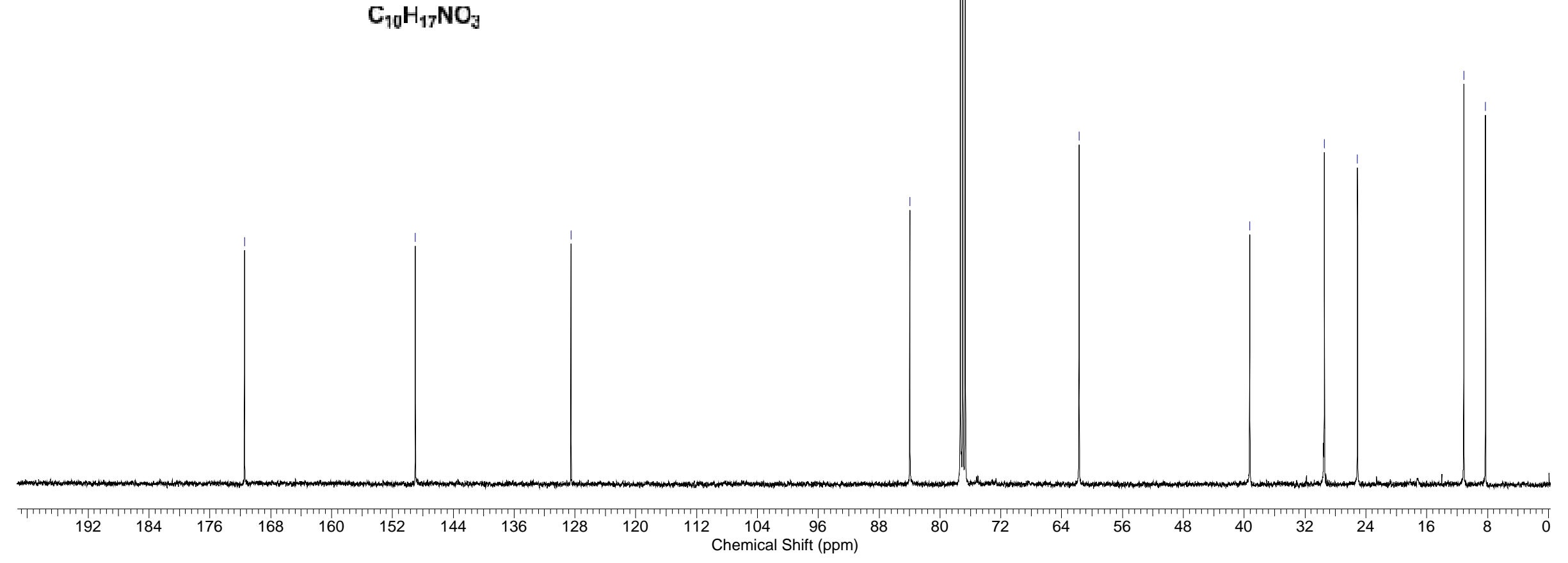




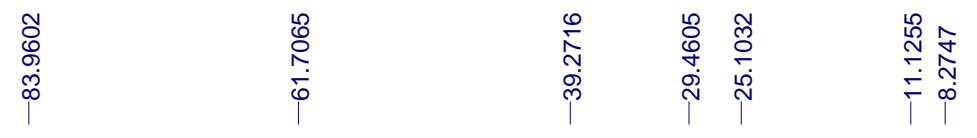

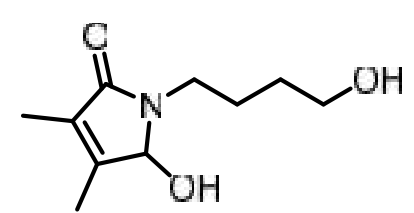

$\mathrm{C}_{10} \mathrm{H}_{17} \mathrm{NO}_{3}$

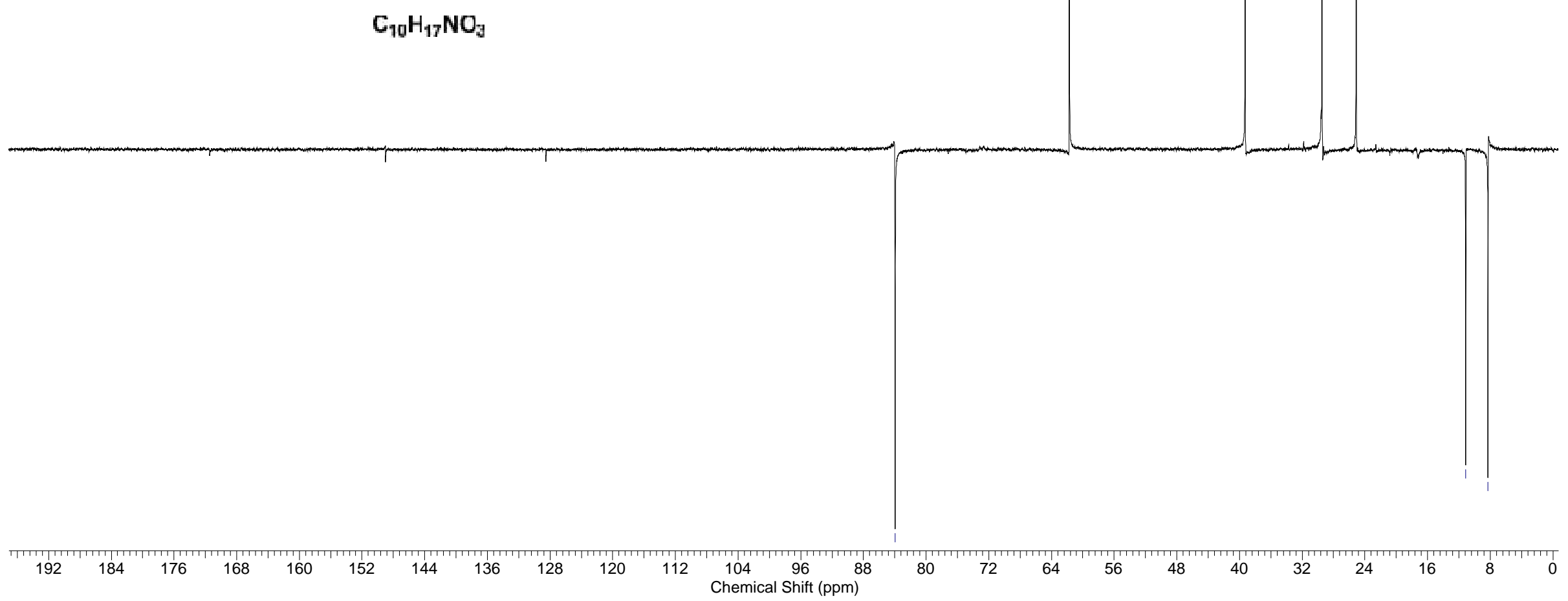




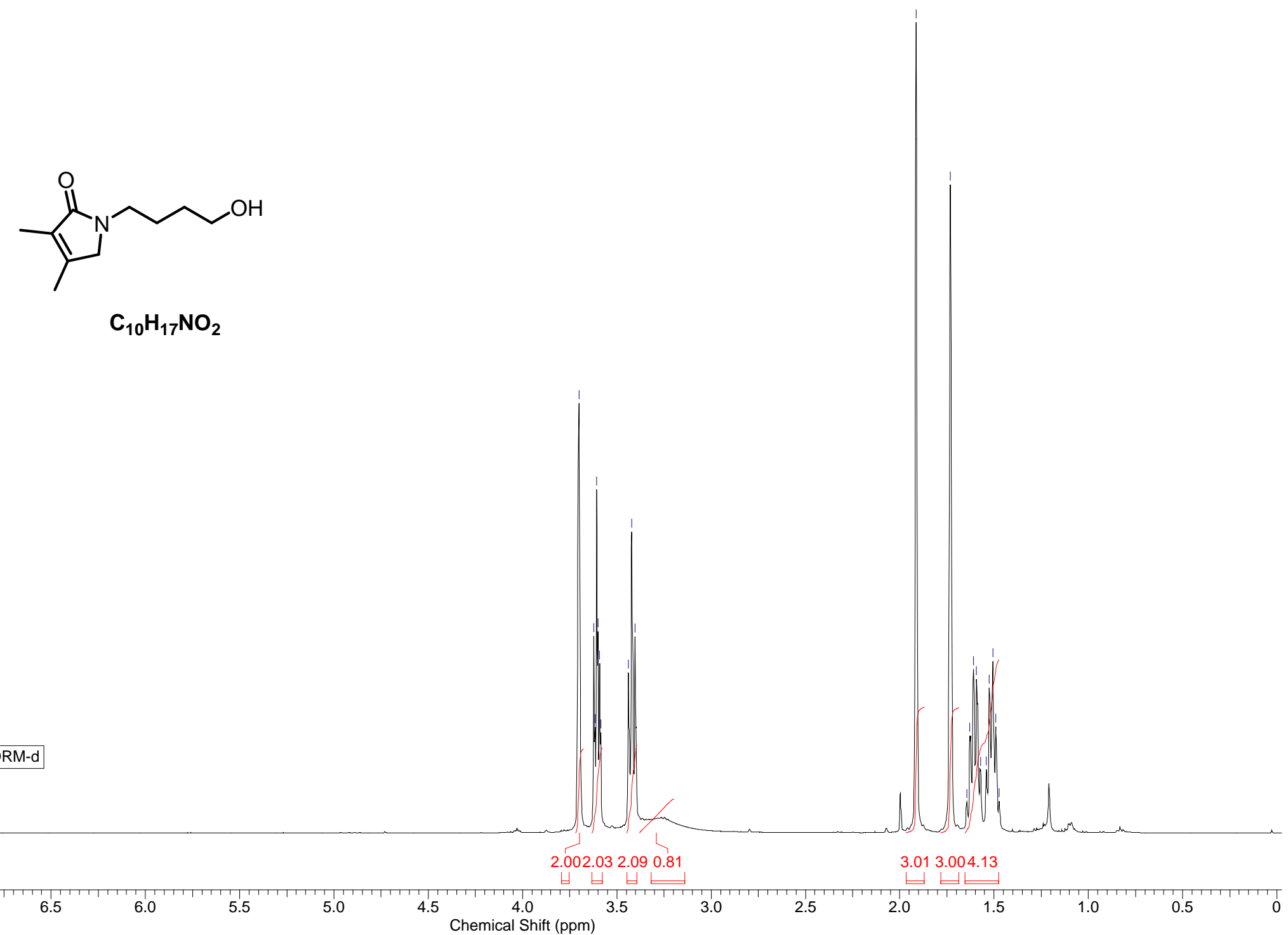


<smiles>CC1=C(C)C(=O)N(CCCCO)C1</smiles>

$\mathrm{C}_{10} \mathrm{H}_{17} \mathrm{NO}_{2}$

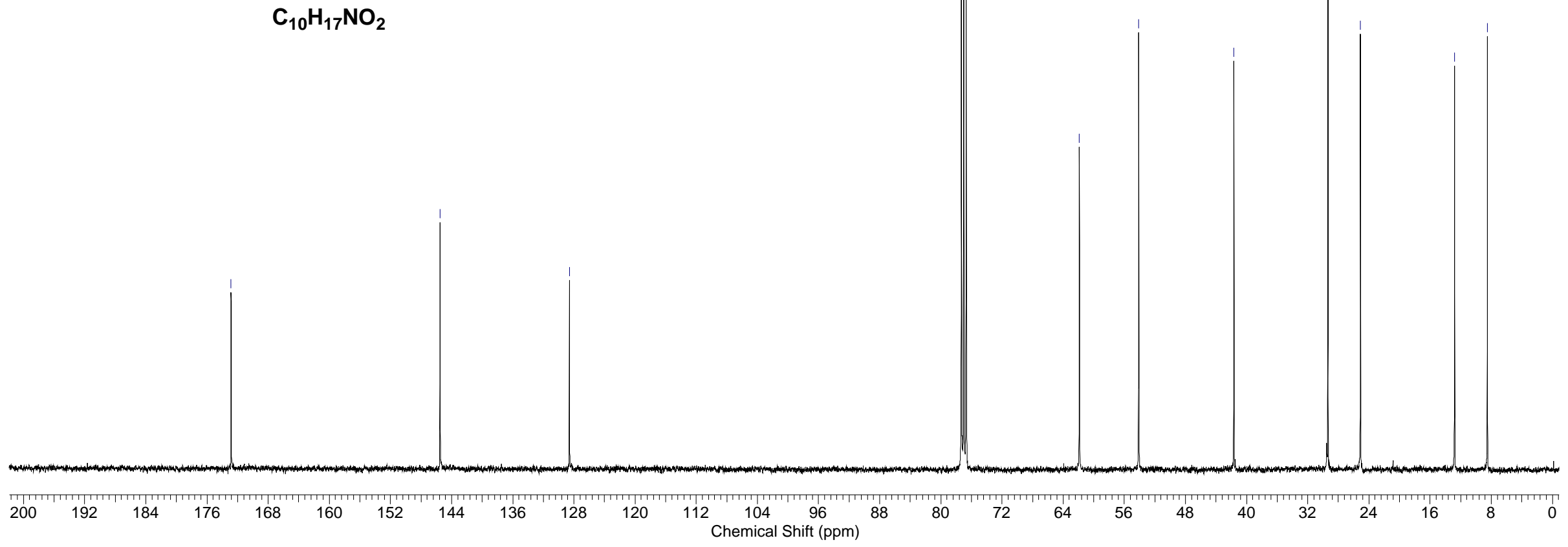




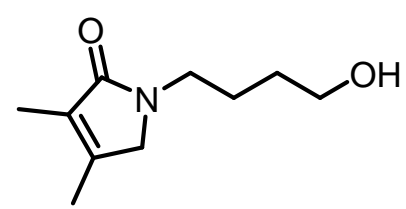

$\mathrm{C}_{10} \mathrm{H}_{17} \mathrm{NO}_{2}$

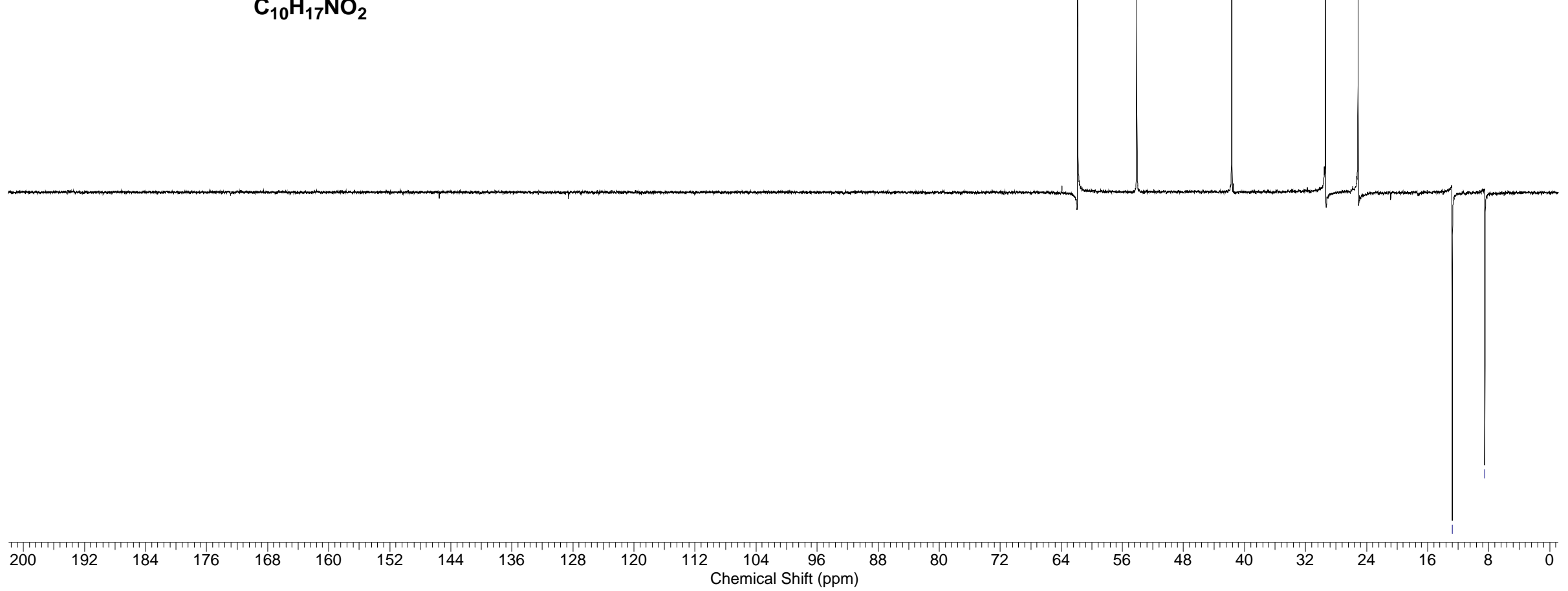




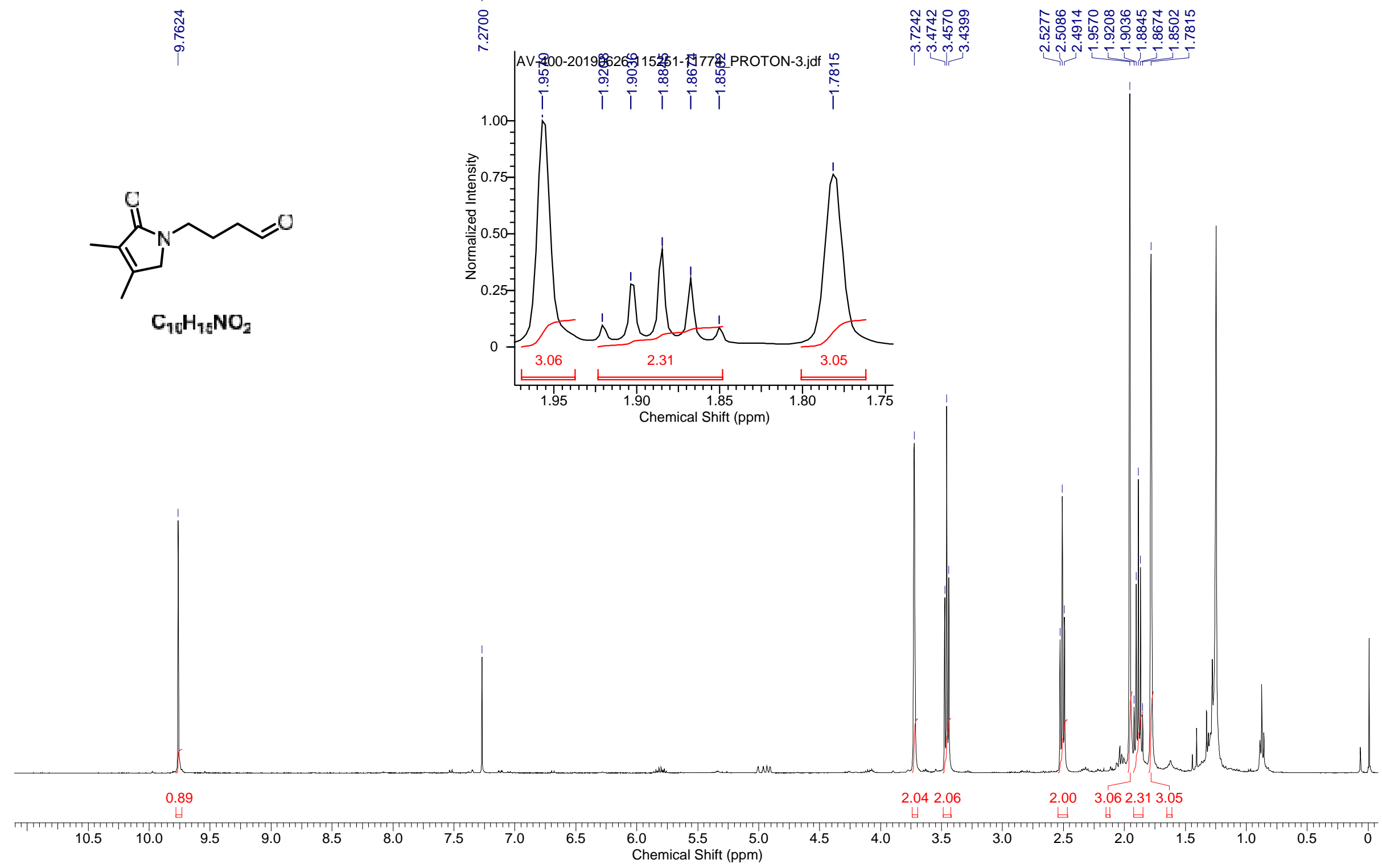



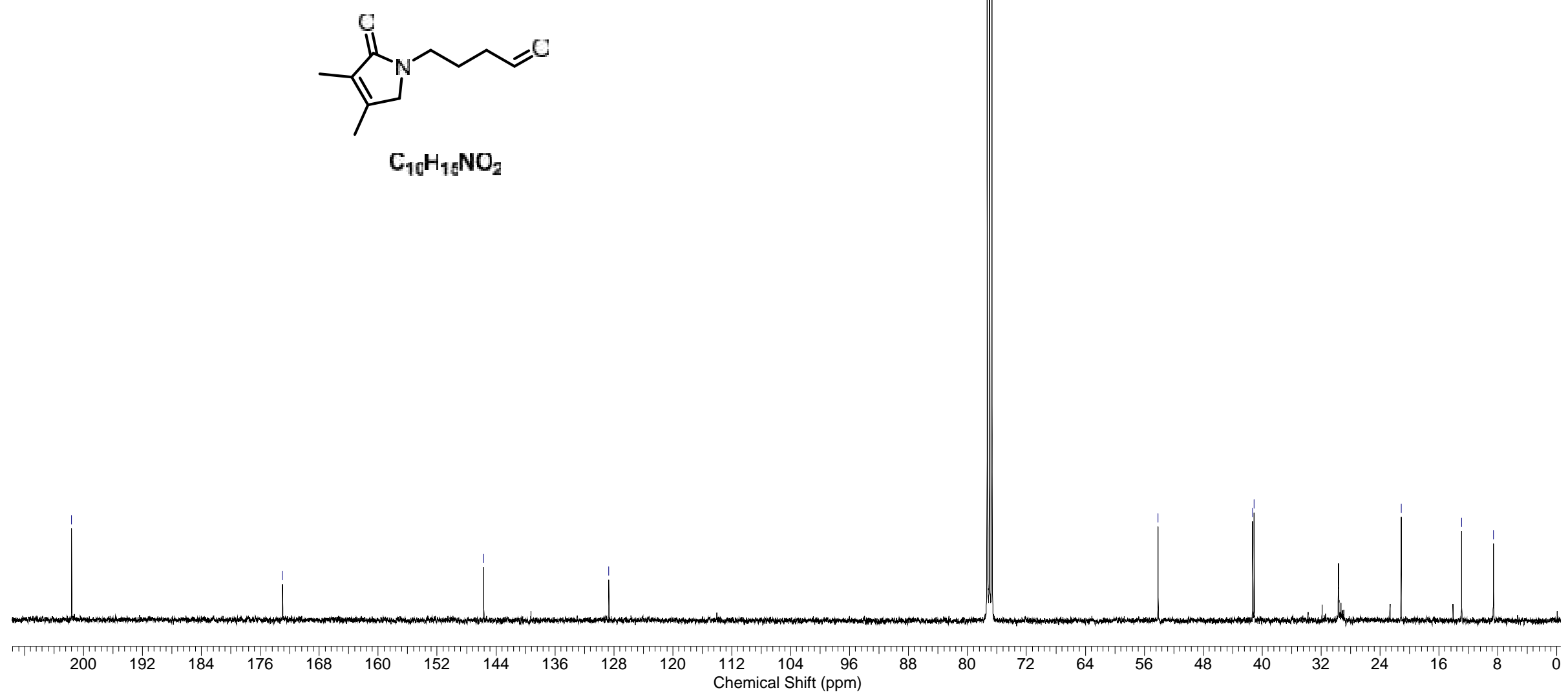

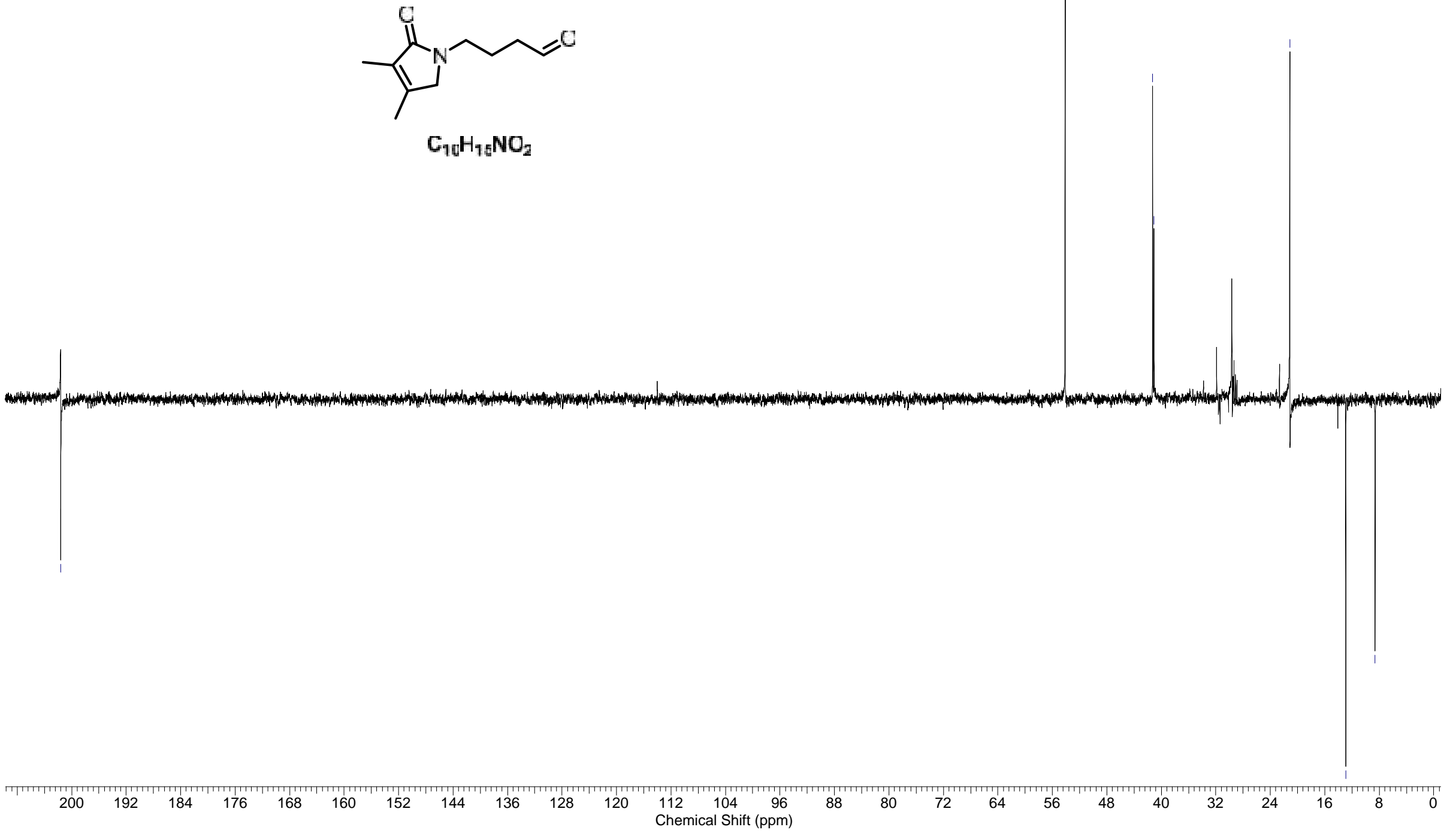


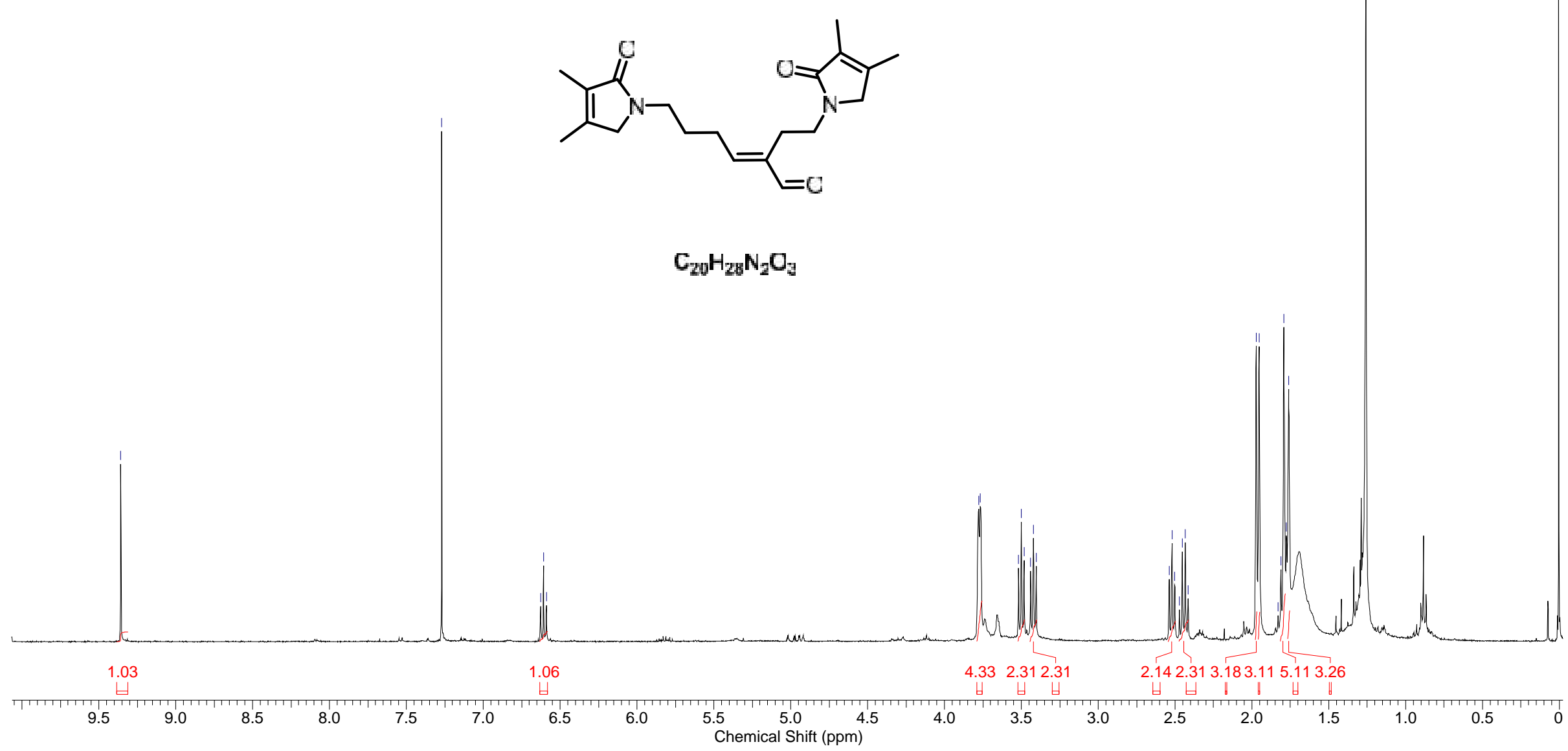

$\mathrm{C}_{20} \mathrm{H}_{28} \mathrm{~N}_{2} \mathrm{O}_{3}$ 

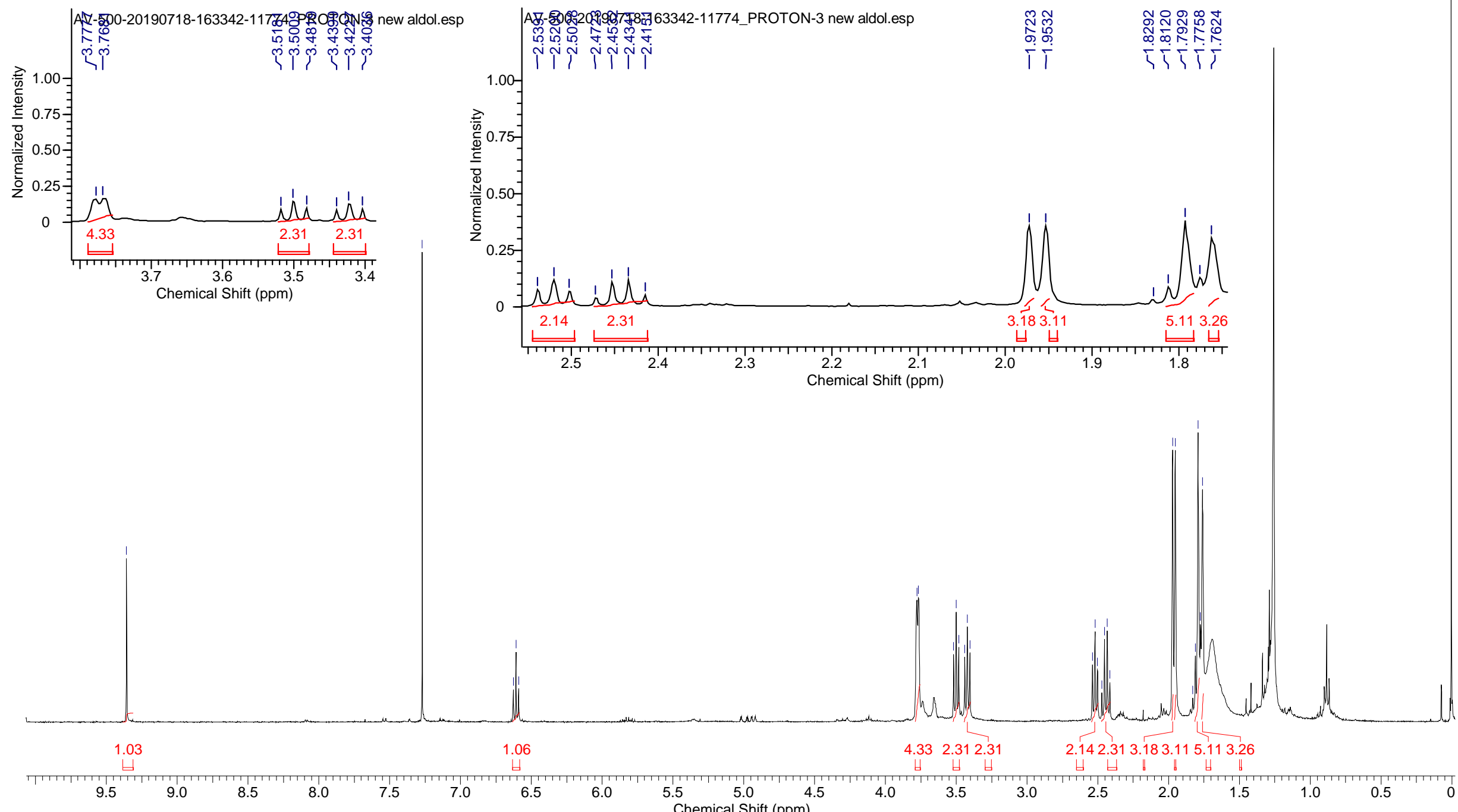


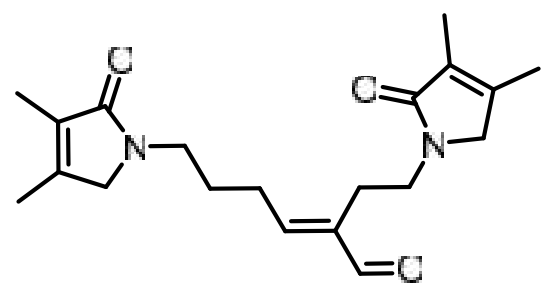

$\mathrm{C}_{20} \mathrm{H}_{28} \mathrm{~N}_{2} \mathrm{O}_{3}$ 


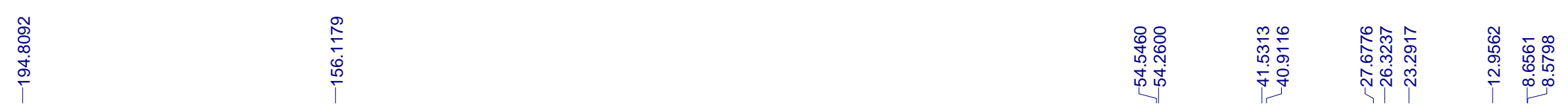

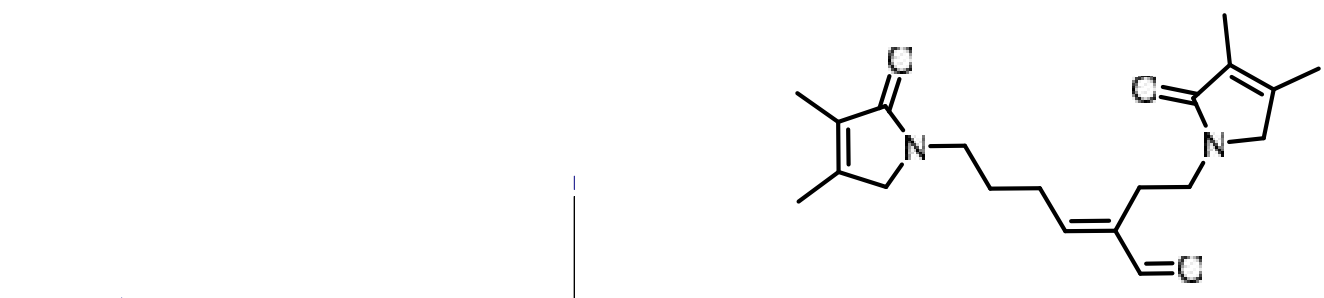

$\mathrm{C}_{20} \mathrm{H}_{28} \mathrm{~N}_{2} \mathrm{O}_{3}$

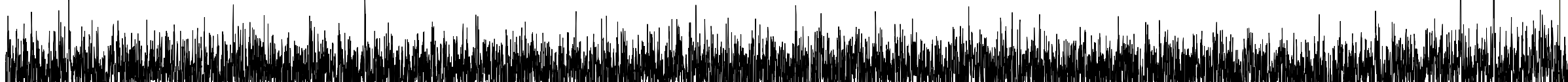

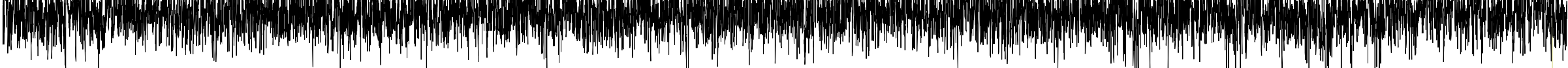

$0 \quad 152 \quad 144$

$136 \quad 128$

120

$1210496 \quad 8896$

S28

Figure S26 : DEPT NMR spectrum of compound 7b 